\title{
Covid-19 et communication de crise. Focus linguistique sur les tweets francophones de Belgique
}

\author{
Louise-Amélie COUGNON \\ Université catholique de Louvain \\ Institut Langage et Communication \\ Media Innovation and Intelligibility Lab \\ Ruelle de la lanterne magique, 14 B-1348 Louvain-la-Neuve \\ louise-amelie.cougnon@uclouvain.be
}

\section{Louis DE VIRON}

DataText SPRL

louis@datatext.eu

The Covid-19 health crisis of 2020 strongly affected the international community. Especially during the lockdown period, social media were widely used for information and emotion sharing. This article aims to keep a material trace of these crisis communication trends. To reach this aim, we applied quantitative and qualitative methods on a corpus of 100,000 tweets we collected in the French-speaking part of Belgium. The corpus is divided into three sub-groups: citizens, politicians and the media. We first present the corpus collection and the methodology we followed. We also look at the lexical creativity that resulted from the crisis and the lockdown situation. We then propose a semantic approach of the themes that emerged from the crisis tweets and which highlight citizens' concerns. Finally, we depict the personalities related to the crisis, by focusing on their communication and on the image they portray to the public.

Keywords:

COVID-19, Crisis Response, Twitter, Discourse, Neology, Population, Politics, Media

Mots-clés:

Covid-19, réponse de crise, Twitter, discours, néologie, population, politiques, médias

\section{Introduction}

La crise sanitaire vécue en 2020 en raison de la pandémie de coronavirus (Covid-19) a affecté la communauté internationale de manière soudaine et durable. Face aux taux d'infection et de mortalité élevés relevés dès l'apparition du virus en Chine en décembre 2019, les gouvernements nationaux ont plus ou moins rapidement implémenté une série de mesures visant à ralentir la dissémination et l'impact du virus sur leur territoire. En Belgique, un confinement strict a été décrété à la mi-mars 2020, imposant la fermeture des commerces non-essentiels, encourageant fortement la pratique du télétravail, et réduisant au strict minimum les contacts familiaux et sociaux. II est vite apparu que l'épidémie et la situation de confinement engendraient un nombre important de discours officiels, de questionnements et de critiques de la population, dont les réseaux sociaux se sont rapidement fait l'écho. 
(1) Il faut le DIRE et le REDIRE à ceux qui sont revenus d'Italie, même si c'est déjà fort tard: restez chez vous jusqu'à 2 semaines après votre retour. N'attendez pas que Maggie De Block se réveille. \#COVID19be (6 mars 2020)

D'un côté, les responsables politiques ont implémenté des mesures plus ou moins drastiques de distanciation sociale et, de l'autre, la population a partagé de l'information et exprimé son opinion via des plateformes de réseautage social telles que Twitter. Plusieurs études ont mis en évidence que la pandémie autant que le confinement ont accru les niveau d'anxiété des citoyens (Lits et al. 2020). Ce sentiment s'est également répandu sur les réseaux sociaux.

(2) Conversation avec moi-même tous les matins depuis le début du confinement. - Je vais bien aujourd'hui mais j'ai peut-être déjà le coronavirus sans le savoir. C'est flippant (29 mars 2020)

L'utilisation de la CéMO (Communication écrite médiée par ordinateur, Cougnon \& François 2011), et en particulier des réseaux sociaux, comme vecteurs d'opinion et outils de dialogue, s'est d'ailleurs quelque peu amplifiée en raison même de la distanciation sociale (Lopez et al. 2020) et de la crise, puisque les médias sociaux offrent un outil de communication rapide ("real-time information", Hoste et al. 2016) et direct (Utz et al. 2013). Collecter l'historique de ces discours afin de garder une trace mémorielle matérielle de la communication de crise de 2020 était alors notre premier objectif.

Les situations de crise ont fait l'objet de nombreuses recherches, portant principalement sur les crises sanitaires, écologiques et des organisations. La majeure partie de ces études porte sur la façon de gérer la crise (Crisis communication, CC: Holtzhousen \& Zerfass 2015) et d'y répondre (Crisis Response, CR: Fediuk et al. 2010), ce qui a abouti à l'établissement de modèles de réponse de crise (Crisis Communication Models, CCM et Situational Crisis Communication Theory, SCCT: Coombs 2012), portant souvent sur des aspects linguistiques des messages échangés durant ces situations (Rachfał 2016; Borden \& Zhang 2019), voire même des modèles appliqués aux réseaux sociaux dans leurs spécificités (social mediated crisis communication model, SCCM: Austin et al. 2012).

La situation de crise du Covid-19 étant récente, nous proposons une étude exploratoire des aspects linguistiques de cette crise: comment elle est nommée, à quoi elle se réfère, ce qu'en pensent et ce qu'en disent la population, les médias et les politiques, et comment les acteurs de la crise sont perçus durant celle-ci. Au cours du prochain chapitre, nous revenons sur la constitution du corpus et les méthodes d'analyse qui lui ont été appliquées. Le chapitre 3 se focalise sur les aspects linguistiques formels permettant de décrire la situation de crise liée au Covid-19, et la créativité lexicale qui en découle. Le chapitre 4 complète cet aperçu formel en adoptant une approche sémantique des référents liés à la situation. Enfin, le chapitre 5 présente les personnalités liées à la situation de crise, l'image qu'elles renvoient dans la population et les 
spécificités de leurs discours propres. Nous concluons cette étude par une discussion des résultats obtenus, suivie d'une description des limites de l'étude et d'une mise en perspective des pistes de poursuite de notre recherche.

\section{Corpus et méthodologie d'analyse}

Ce projet de recherche mené depuis mars 2020 a bénéficié d'une méthode de collecte et d'une méthodologie d'analyse construites précédemment dans le cadre du projet belge Opinio ${ }^{1}$. Cette expertise nous a permis d'être réactifs face au caractère subit de la crise du Covid-19 dans le monde. Nous revenons succinctement sur la description de cette méthodologie et nous présentons également les adaptations qui ont été nécessaires pour coïncider avec la thématique de la crise du coronavirus de 2020.

\subsection{Corpus de messages Twitter}

Le choix de collecter des corpus provenant de Twitter est basé sur de nombreux résultats de recherche en linguistique et en communication, qui ont montré que ce réseau social est très populaire pour rechercher et diffuser de l'information relative aux situations de crise (Hoste et al. 2016; Veil et al. 2011).

La constitution de notre corpus est basée sur un système d'extraction de tweets à travers une API mise à disposition par Twitter ${ }^{2}$. Le système d'extraction pour ce projet se base sur les mots-clés suivants: covid ${ }^{*}$, corona ${ }^{*}$, ncov ${ }^{*}$, sars ${ }^{*}$ et sras* $^{*}$ L'astérisque marque la variabilité possible après la dernière lettre indiquée et la casse n'est pas prise en compte dans cette recherche. La collecte a débuté en mars $2020^{3}$, mais inclut des données depuis janvier 2020 et jusqu'à la fin du mois de mai 2020.

Dans le cadre de notre recherche, un ensemble de comptes Twitter a été préalablement ciblé, correspondant à 3 sous-groupes de la population belge francophone: les citoyens, les politiques et les médias.

\subsubsection{Le corpus citoyen}

Nous nous intéressons aux discours des citoyens dans la mesure où, comme le soulignent Borden \& Zhang (2019), on voit émerger le rôle actif du public dans la création (publication), la réponse (commentaire) et la diffusion (partage) de l'information en temps de crise.

Nous avons collecté un corpus de 72.000 tweets relatifs au Covid-19 provenant de comptes représentant la population belge francophone sur Twitter. Cette représentativité n'est pas à comprendre au sens statistique, mais plutôt en termes d'hétérogénéité des comptes Twitter afin d'atteindre des franges socio-

Projet de la Fédération Wallonie-Bruxelles mené depuis 2018 en Belgique

Ressource disponible sur: https://developer.twitter.com/en/docs

Nous tenons à remercier le Cental pour son aide précieuse dans la collecte des données. 
démographiquement variées de la population de followers. Pour atteindre cette hétérogénéité, nous avons développé une méthodologie de sélection et de filtrage ${ }^{4}$ basée sur les followers de comptes célèbres en Belgique francophone:

- Hommes, femmes et partis politiques

- Sportifs

- Journalistes et médias

- Industries, institutions, organismes et forces de l'ordre (police, armée...)

- Autres célébrités: artistes, royauté, Miss Belgique, youtubeurs...

\subsubsection{Le corpus politique}

La corpus politique est composé de 2.305 tweets abordant le Covid-19. Afin de constituer le corpus le plus représentatif possible de la classe politique belge francophone s'exprimant sur Twitter, nous avons pris en compte une liste de 5 partis politiques disposant d'élus au Parlement fédéral5:

- Le PTB (Parti du Travail de Belgique) est un parti considéré à l'extrême gauche du spectre politique.

- Le PS (Parti Socialiste) est le parti traditionnel de la gauche.

- ecolo (Écologistes confédérés pour l'Organisation de Luttes originales) est un parti dont le principal objectif est de réduire l'empreinte écologique de la Belgique. II est usuellement situé au centre.

- Le cdH (centre démocrate Humaniste) est un parti considéré comme centriste, dont l'histoire est liée au Parti Social Chrétien (PSC).

- DéFI (Démocrate fédéraliste indépendant) est un parti historiquement relié à la défense des francophones de Belgique. II est d'ordinaire placé au centre de l'échiquier politique.

- Le MR (Mouvement Réformateur), parti libéral de droite et centre droit.

Nous avons ensuite sélectionné 50 comptes par parti en commençant par le compte Twitter du parti lui-même, ainsi que les comptes de son président et vice-président. Ensuite, nous nous sommes focalisés sur les élus de la Chambre et du Sénat, du Parlement wallon, de la Région Bruxelles-Capitale, de la Fédération Wallonie-Bruxelles et les élus européens. Puis, si les 50

$4 \quad$ La méthodologie d'extraction, de filtrage et de sélection précise à partir de cette liste a été élaborée en partenariat avec le Cental (UClouvain) dans le cadre du projet Opinio et ne peut être partagée publiquement.

5 Pour établir cette liste, nous avons choisi de nous baser sur une source officielle, le site Belgium.be, une initiative des autorités fédérales, développé et entretenu par le SPF Chancellerie du Premier Ministre et le SPF Fedict, en étroite collaboration avec l'ensemble des services publics fédéraux, et avec les services publics régionaux et communautaires. 
comptes n'étaient pas encore listés, nous avons cherché des comptes d'élus locaux. La productivité de tweets Covid-19 par parti est très variable; la Fig. 1 présente la répartition par parti des tweets ayant trait à cette thématique. On observe que seuls 126 comptes de politiciens sur 250 ont été productifs sur ce sujet durant la période étudiée, produisant quelques 2.305 messages.

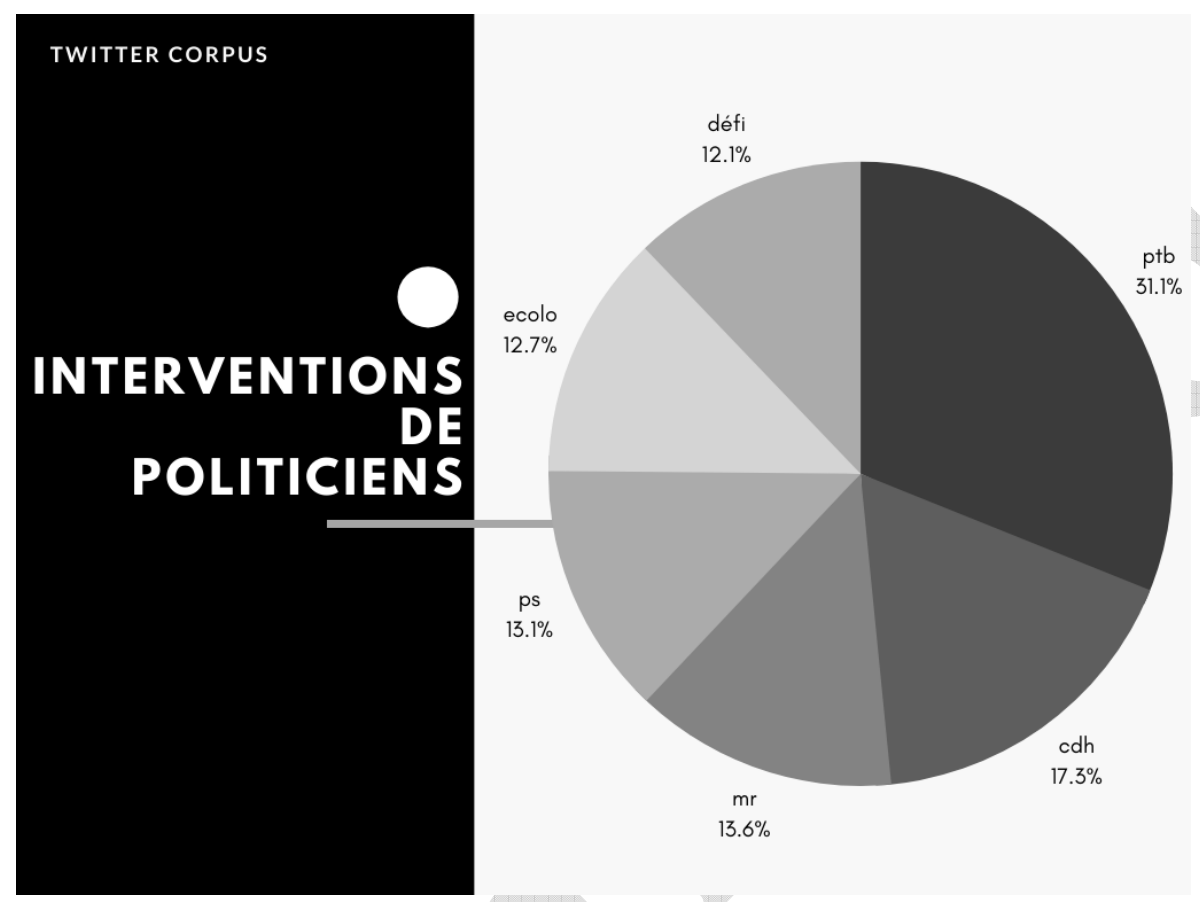

Fig. 1 Taux d'intervention sur Twitter de partis politiques belges francophones au sujet du Covid-19 durant la période du confinement (source: Cougnon \& de Viron, 2020).

La productivité des personnalités politiques est également très peu homogène. La Fig. 2 présente les 15 comptes les plus prolixes au sujet du Covid-19.

Fig. 2 Classement des comptes de personnalités politiques belges francophones les plus prolixes à

\begin{tabular}{llrrlr} 
Pos & \multicolumn{1}{c}{ Nom } & $\begin{array}{c}\text { Nb } \\
\text { tweets }\end{array}$ & Pos & \multicolumn{1}{c}{ Nom } & $\begin{array}{c}\text { Nb } \\
\text { tweets }\end{array}$ \\
$\mathbf{1}$ & Germain Mugemangango (PTB) & $\mathbf{2 0 2}$ & $\mathbf{9}$ & Saskia Bricmont (ecolo) & 72 \\
$\mathbf{2}$ & Catherine Fonck (cdH) & 157 & $\mathbf{1 0}$ & François Desquesnes (cdH) & 68 \\
$\mathbf{3}$ & Denis Ducarme (MR) & 149 & $\mathbf{1 1}$ & Sophie Wilmès (MR) & 54 \\
$\mathbf{4}$ & Sofie Merckx (PTB) & 138 & $\mathbf{1 2}$ & Pierre-Yves Jeholet (MR) & 54 \\
$\mathbf{5}$ & Elio Di Rupo (PS) & 131 & $\mathbf{1 3}$ & Georges-L Bouchez (MR) & 47 \\
$\mathbf{6}$ & Didier Reynders (MR) & 97 & $\mathbf{1 4}$ & Alain Maron (ecolo) & 44 \\
$\mathbf{7}$ & Philippe Close (PS) & 93 & $\mathbf{1 5}$ & Rudi Vervoort (PS) & 38 \\
$\mathbf{8}$ & Julie Fernandez (PS) & $\mathbf{8 7}$ & & &
\end{tabular}

propos du Covid-19 sur Twitter par nombre de tweets émis durant la période de crise. 


\subsubsection{Le corpus médiatique}

Nous avons également collecté un corpus médiatique, dans la mesure où les médias s'avèrent essentiels en temps de crise, comme sources d'information, mais aussi comme gestionnaires de ressources, par exemple lorsqu'ils permettent la coordination de l'appel à l'aide et à la participation (Veil 2009).

Le corpus médias comporte 33.000 tweets qui abordent le Covid-19. II est constitué de tweets provenant de 24 comptes d'information francophone belge. Parmi ceux-ci, on retrouve:

- les grands médias d'information, tels que RTBF, rtlinfo, LeVif, lesoir, sudpresseonline, lecho, ladh, lalibrebe, L'Avenir et LesNews24;

- les médias régionaux, tels que LaMeuse_be et DHBruxelles;

- les médias spécialisés, tels que ParisMatchBe et RTLsportbe;

- les versions francophones de médias néerlandophones, tel que VRT_Flandreinfo;

- et la presse subjective, tel que infopunaises.

La productivité de ces médias sur Twitter est également variable; nous fournissons dans la Fig. 3 un aperçu des comptes les plus productifs.

\begin{tabular}{|l|l|l|l|l|}
\hline account & name & tweets & mentions & partages (urls) \\
\hline RTBFinfo & RTBF info & 5969 & 2905 & 9551 \\
\hline sudpresseonline & Sudpresse & 6445 & 2384 & 3222 \\
\hline lesoir & Le Soir & 2991 & 1267 & 6804 \\
\hline LeVif & LEVIF / L'EXPRESS & 1188 & 925 & 2535 \\
\hline rtlinfo & RTL info & 1688 & 911 & 1790 \\
\hline lavenir_net & lavenir.net & 1508 & 644 & 1533 \\
\hline lalibrebe & lalibre.be & 3603 & 360 & 3941 \\
\hline lecho & L'Echo & 2710 & 304 & 1224 \\
\hline ladh & DH les Sports + & 3289 & 181 & 1760 \\
\hline Belgique_info & Belgique Info & 793 & 4 & 0 \\
\hline
\end{tabular}

Fig. 3 Classement des 10 médias d'information les plus populaires auprès de la population belge francophone durant le confinement provoqué par la pandémie de Covid-19 en 2020. 
La popularité des médias est ici calculée sur la base du nombre d'urls dans les tweets de la population qui pointent vers leur site web. Ce chiffre est accompagné du nombre de mentions (@) de ces médias dans les tweets, qui constitue également un bon indicateur: nous voyons en effet que la colonne "mentions" suit la même courbe de classement que la colonne "partages". Enfin, la colonne "tweets" précise le nombre de tweets produits par ce média pendant la même période: on voit qu'elle ne suit pas la même courbe, ce qui permet d'invalider un lien direct entre popularité et production de contenu.

\subsection{Méthodes d'analyse combinées}

Un nombre croissant d'ouvrages, tel que Holtzhousen \& Zerfass (2015), ont mis en avant la nécessité d'élargir la connaissance de la $\mathrm{CC}$ en ayant recours à une perspective purement linguistique. Suivant le modèle CCM, nous avons donc choisi des méthodes qui permettent d'extraire à partir des tweets des traits linguistiques portant sur la communication sur le Covid-19, sur la réaction à la crise et sur la réputation de ses acteurs majeurs (Utz et al., 2013). Pour mener à bien ce projet, des méthodes qualitatives et quantitatives ont été croisées.

Les analyses lexicométriques des noms communs ont été menées à l'aide du dictionnaire Delaf (dictionnaire des formes fléchies du français, Courtois 2004), pour rechercher les formes fléchies d'un lemme et augmenter la couverture d'extraction des mots. Pour les formes inconnues du Delaf, comme les néologismes, nous les avons fléchies manuellement avant de les rechercher à l'aide d'expressions régulières. La détection des entités nommées a été réalisée par French TwitlE, un outil développé par Gate, et spécialisé dans l'extraction d'information dans les tweets en français (Derczynski \& al. 2016). La tokenization, l'analyse POS, de dépendance sujet-verbe et l'extraction des expressions multi-mots ont été réalisées en Python avec la librairie Spacy (Honnibal \& Montani 2017).

L'analyse de spécificités, basée sur la comparaison avec un corpus d'exclusion, a permis de détecter des expressions suremployées dans un sous-corpus de messages par rapport à l'ensemble des tweets collectés. Cette méthode permet notamment de dégager les stratégies de communication et des habitudes langagières pour une catégorie de population ou à un moment précis de la crise. Pour étudier ces phénomènes, nous avons utilisé une approche lexicométrique analysant la distribution des probabilités hypergéométriques des mots et multimots au sein du corpus (Lebart \& Salem 1994).

Pour les analyses thématiques et de polarité, nous avons combiné des méthodes d'extraction automatiques, telles que définies ci-dessous, avec un système d'annotation manuelle à un seul annotateur. L'évolution des tendances 
à travers le temps a été rendue possible grâce au logiciel de visualisation Tableau Public Edition $2020^{6}$.

\section{Covid-19 et créativité lexicale}

Comme l'a montré Caldas (2015), tout contexte de crise amène un processus d'innovation lexicale et terminologique. Dans le cas de la pandémie, la dénomination même du virus a également fait l'objet de questionnements, ce qui s'est traduit dans les tweets de la population belge francophone.

(3) Le \#coronavirus est apparu en Chine, pourquoi il ne porte pas un nom chinois? (29 janvier 2020)

Comme expliqué précédemment, afin de nous donner une idée globale de cette dénomination, nous avons enquêté sur les variétés ${ }^{7}$ de forme de "covid"", de "coronavirus", de "ncov", de "sars" et de "sras" utilisées dans les tweets sur toute la période du confinement ${ }^{8}$. La Fig. 4 résume la répartition des variétés pour l'ensemble du corpus de tweets et par corpus spécifique.

\begin{tabular}{|l|l|l|l|l|}
\hline Variante & Fréq. totale & Politique & Médias & Population \\
\hline coronavirus & $80776(0.71)$ & $1275(0.56)$ & $31594(0.88)$ & $47907(0.63)$ \\
\hline covid19 & $19232(0.17)$ & $826(0.36)$ & $1379(0.04)$ & $17027(0.22)$ \\
\hline covid-19 & $11985(0.11)$ & $152(0.07)$ & $2598(0.07)$ & $9235(0.12)$ \\
\hline corona & $1128(0.01)$ & $12(0.01)$ & $83(0)$ & $1033(0.01)$ \\
\hline covid & $565(0)$ & $5(0)$ & $63(0)$ & $497(0)$ \\
\hline sars-cov-2 & $151(0)$ & $0(0)$ & $4(0)$ & $147(0)$ \\
\hline covid 19 & $76(0)$ & $1(0)$ & $6(0)$ & $69(0)$ \\
\hline 2019-nCov & $44(0)$ & $0(0)$ & $2(0)$ & $42(0)$ \\
\hline sras-cov-2 & $11(0)$ & $1(0)$ & $1(0)$ & $9(0)$ \\
\hline
\end{tabular}

Fig. 4 Fréquence des variantes du mot "coronavirus" utilisées dans le corpus de tweets de Belgique francophone durant le confinement. Le nombre d'occurrences est suivi du pourcentage.

Les formes "coronavirus" (71\%), "covid19" (17\%) et "covid-19" (11\%) sont majoritairement employées par l'ensemble de la population enquêtée, bien que nous notions une prévalence, par rapport à la moyenne de la population de la forme "coronavirus" par les médias, de la forme "covid19" par les personnalités politiques et de la forme "Covid-19" par les citoyens. Les variétés "ncov", "sars" et "sras" sont quant à elles sous-employées, toutes populations confondues, probablement car plus complexes à énoncer et à rédiger. On note un faible

$6 \quad$ Lien vers le logiciel: https://public.tableau.com/

Ces variétés incluent autant les forms fléchies de ces racines que les hashtags de ces formes.

Nous avons aussi enquêté sur d'autres formes ne provenant pas de ces racines, mais leur fréquence était négligeable. 
emploi de la forme simple "covid 19" sans le trait d'union, ce qui va à l'encontre de la tendance propre à la CéMO: soudure des mots composés et chute des accents et des traits d'union (Cougnon et al. 2013). Ceci s'explique sans doute par la forme simplifiée compétitive "covid19" qui a remporté le suffrage, surtout auprès des autorités politiques, ainsi que par le fait que le hashtag s'y référant n'accepte pas l'espace.

La Francophonie a également vu naitre un débat sur le genre attribué au mot "covid". En effet, si "coronavirus" a depuis plus de 50 ans hérité du genre du mot "virus", c'est-à-dire masculin, le cas de "covid-19" a fait débat dans les médias et au sein de la population, principalement à partir de l'annonce de l'Académie française de "préférer"9 la forme féminine "la covid", attribuant le genre du nom qui constitue le noyau du syntagme (disease, traduit par le substantif féminin maladie) comme c'est le cas pour d'autres acronymes, fonctionnant ainsi sur une règle historique, plutôt que sur l'usage.

(4) Doit-on dire "le" ou "la" Covid-19 ? Selon l'Académie française, le féminin est "préférable" (8 mai 2020)

La Fig. 5 présente la répartition du genre du mot "covid" au sein de la population belge francophone: $98,4 \%$ des tweets $(\mathrm{N}=2.671)$ des citoyens citant le mot "Covid-19" utilisent la forme masculine. Nous notons en outre qu'aucun des tweets politiques sur cette thématique ne comprend la forme féminine. Au niveau des médias belges, nous ne remarquons pas de changement de tendance sur le genre ( $1 \%$ des occurrences sont au féminin).

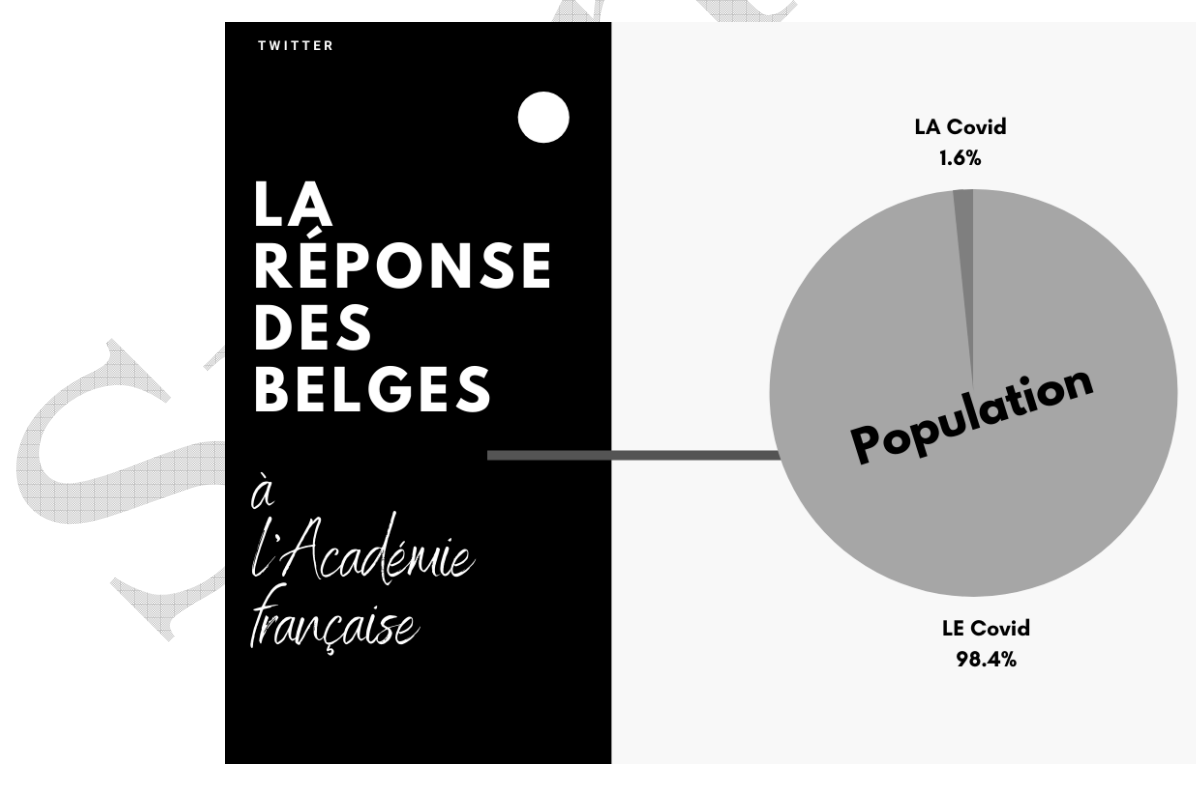

$9 \quad$ Et l'Académie complète: "pour redonner à cet acronyme le genre qui devrait être le sien", remplaçant ainsi le conseil par la norme (http://www.academie-francaise.fr/le-covid-19-ou-lacovid-19). 
Fig. 5 Répartition du genre du mot "covid" au sein des tweets collectés auprès de la population belge francophone (source: Cougnon \& de Viron, 2020).

Après avoir étudié la "morphologie" du lemme coronavirus et son genre, nous regardons maintenant de plus près les néologismes formels et sémantiques qui sont apparus durant la période du confinement. Tout d'abord, nous avons découvert l'utilisation massive de la forme verbale "réouvrir", en lieu et place de la forme attendue "rouvrir". En effet, le Larousse (2020), par exemple, précise: "Dans l'expression soignée, et en particulier à l'écrit, on emploie rouvrir et non *réouvrir". Or, à l'encontre des recommandations normatives, la forme "réouvrir" est également populaire (cf. Annexe 1) parmi les citoyens, car elle est calquée sur le substantif y référent "réouverture". Le verbe apparait d'ailleurs conjugué à de nombreux temps et modes, comme le montrent les exemples 5 et 6 .

(5) Les parents qui ne mettront pas leurs enfants à l'école lorsque l'on pourra reprendre seront les premiers à l'entrée de Walibi et Bellewaerde quand ils réouvriront. \#covid19 \#Belgique (24 avril 2020)

(6) Au Japon ils ont réouvert les écoles et ils les ont refermée car une centaine de cas en un rien de temps suivez l'exemple pour un mois et demi d'école prendre de telle risque! $2 e$ vague assurée \#coronavirus (22 avril 2020)

C'est principalement face à l'arrivée rapide de termes anglais pour définir la situation internationale de crise que les francophones ont réagi en proposant de nouveaux sens, de nouveaux mots et de nouvelles expressions pour décrire des réalités qu'ils ont découvertes durant les premiers mois de $2020^{10}$. C'est le cas du triplet "confiner" (186 occurrences dans le corpus population) "confinement" (5496 occurrences dans le corpus population) - "confiné" (729 occurrences dans le corpus population), qui est venu s'imposer très rapidement face à "lockdown" (585 occurrences dans le corpus population, dont 27 pour "lockdown party").

(7) Coronavirus: drones et robots coursiers à la rescousse des humains confinés (2 avril 2020)

Le verbe "confiner" existe depuis le $15^{\mathrm{e}}$ siècle $\left(16^{\mathrm{e}}\right.$ pour le substantif "confinement"), mais se rapporte au fait de reléguer quelqu'un dans un certain lieu parce qu'il est prisonnier. Le sens sanitaire utilisé aujourd'hui tient plus au monde des animaux, que l'on doit séparer dans les élevages trop intensifs, ou aux précautions prises pour empêcher la dissémination de produits radioactifs dans les centrales nucléaires (Larousse, 2020).

Le triplet opposé, "déconfinement" (1077 occurrences dans le corpus population) - "déconfiner" (80 occurrences) - "déconfiné" (17 occurrences) ressort également de la créativité lexicale, puisqu'il n'existe encore dans aucun dictionnaire de langue française officiel.

10 Nous remercions le prof. émérite Michel Francard qui, par sa chronique "UCLouvain @ home", nous a fortement inspirés pour la partie sur la néologie. 
(8) Les \#covid19BE \#restezchezvous

En avril, ne te déconfine pas d'un cil ! Ne sortez de chez vous que pour des déplacements essentiels. Pas d'arrêt, pas de pic-nic, pas de regroupements. Rester chez vous = sauver des vies $\boldsymbol{G}_{0}$ Merci à toutes et tous qui poursuivez l'effort jusqu'au bout! (3 avril 2020)

Une autre innovation linguistique de la période de crise Covid-19 réside dans les expressions plus complexes. La plus fréquente ${ }^{11}$ dans notre corpus est l'expression "distanciation sociale" (276 occurrences dans le corpus population) au lieu de "distanciation physique" (19 occurrences). Calque de l'expression anglaise "social distancing" (49 occurrences), la formule "distanciation sociale" a été proposée: c'est un glissement sémantique qui se réalise à partir d'un sens ancien appliqué au théâtre de Brecht (traduction du terme allemand Verfremdungseffekt, "le public est maintenu à distance des événements relatés", Larousse, 2020). Cette expression, utilisée dans les tweets, se réfère en réalité très largement à la "distanciation physique" d'un individu envers un autre, par une série de "gestes barrières" (autre expression nouvelle moins populaire, 37 occurrences dans le corpus population ${ }^{12}$ ), incluant $1,5 \mathrm{~m}$ de distance entre individus (en Belgique), le port du masque et l'éternuement dans le coude, entre autres choses.

(9) J'ai testé 3 boulangeries dans mon quartier: AUCUN employé ne porte de masque, seul 1 parmi eux porte des gants mais prend le pain et ensuite les pièces de monnaie avec ces mêmes gants! \#covid19belgium \#COVID_19 \#gestesbarrières (1 ${ }^{\text {er }}$ mai 2020)

L'expression "distanciation sociale" se réfère explicitement à un type de prise de distance d'un groupe social par rapport à un autre: fermeture des écoles, des lieux de culte, des lieux de travail et d'installations de loisirs. Le risque est de faire prendre à l'expression le sens de "rupture du lien social": certains sociologues du $20^{\mathrm{e}}$ siècle ayant utilisé le terme dans le sens de "refus de se mêler à d'autres classes sociales" (Dumazedier \& Ripert 1966), sens que nous retrouvons justement dans les tweets:

(10) \#Rwanda Les mesures drastiques de confinement ordonnées par \#PaulKagame tuent plus que la pandémie elle-même. \#Kagame applique parfaitement la distanciation sociale en refusant de s'impliquer personnellement auprès de son peuple qui meurt de faim.

Coronavirus 0 - 1 Famine ( ${ }^{\text {er }}$ avril 2020)

(11) Pour se protéger du \#COVID-19, on nous demande de respecter la \#distanciation \#sociale. Pourtant, les riches n'ont pas attendu le \#coronavirus pour ne pas parler aux pauvres (30 mai 2020)

Rappelons encore que la "distanciation" est un processus, alors que la "distance ${ }^{13 "}$ en est le résultat: cette distinction n'est pas anecdotique dans la mesure où les gestes barrières évoqués semblent davantage tenir du contrôle de soi proactif plutôt que d'une situation donnée et immuable.

11 Étonnamment, l'expression cours/travail "en présentiel" est très rare dans le corpus de tweets francophones de Belgique (13 occurrences dans le corpus population).

12 Invariablement au pluriel (pour "gestes") et sans trait d'union dans l'ensemble de notre corpus.

13 On trouve 29 occurrences de l'expression "distance sociale" dans le corpus population. 


\section{Thématiques de la crise}

Les thématiques abordées durant la période du confinement en Belgique francophone ont reflété les préoccupations liées à la peur de la maladie, à la situation de confinement et à la gestion politique de la crise. Si l'on se penche sur l'emploi des variantes de "Covid-19" dans les tweets de janvier à juin 2020 (Fig. 6), on observe une tendance qui se retrouve dans chacun des souscorpus: la progressive incursion du sujet Covid-19 dans les tweets, jusqu'à un pic coïncidant avec le début des mesures de confinement (mi-mars), momentclé de la communication d'information et du questionnement. Ce pic est suivi d'une diminution du nombre de tweets jusqu'à la phase 3 du déconfinement, le 8 juin 2020, qui correspond à la réouverture partielle du secteur de l'Horeca ${ }^{14}$ et à la possibilité de se "re-sociabiliser" en fréquentant des groupes de 10 personnes par semaine. Ces nouvelles mesures ont engendré une large diversification des thématiques des messages.

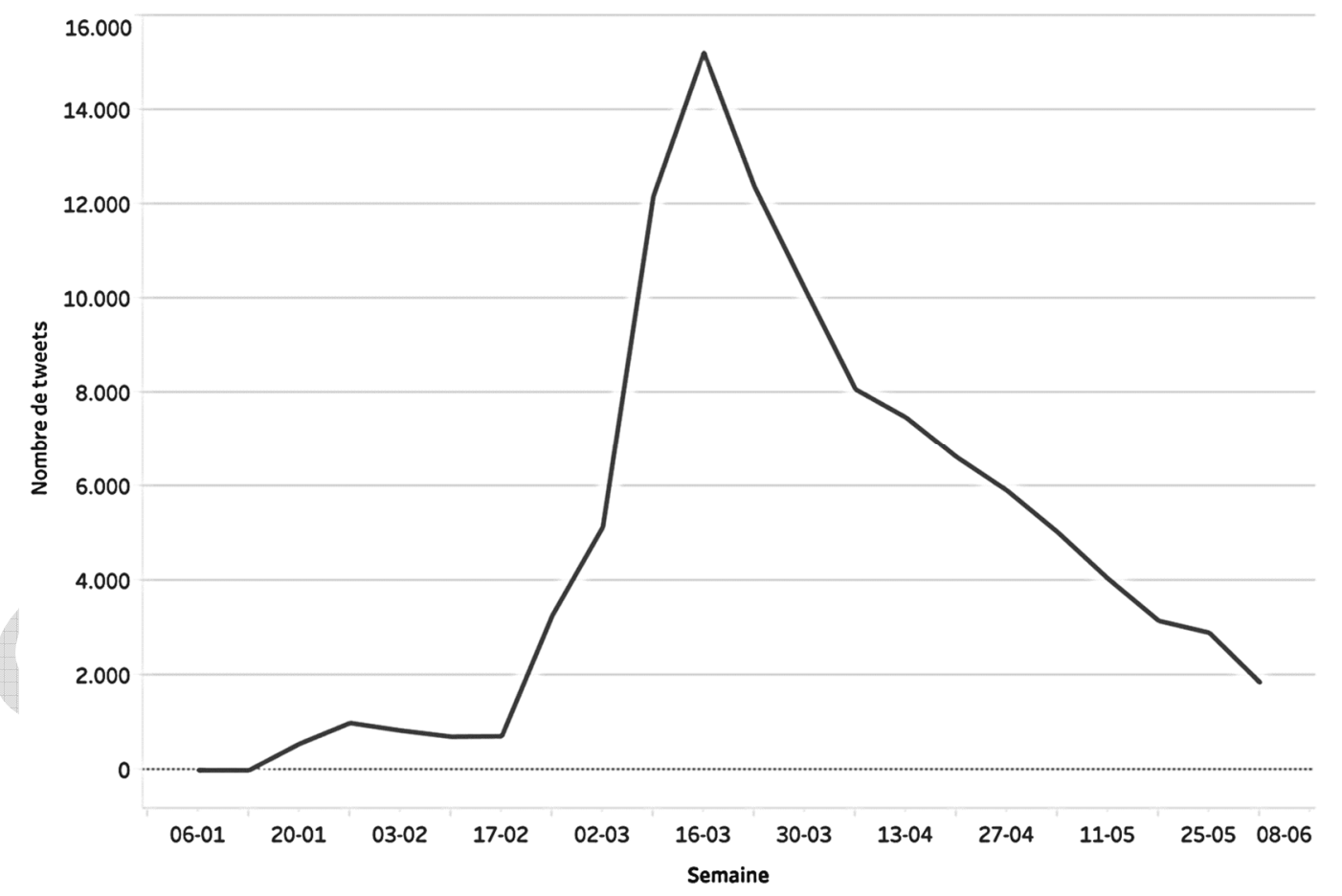

Fig. 6. Fréquence d'apparition des expressions "covid" au sein des tweets collectés auprès de la population belge francophone durant la période de confinement.

14 En Belgique, le secteur de l'Horeca regroupe les hôtels, restaurants et cafés, ce compris les boites de nuit, ces dernières n'ayant pas eu la permission de rouvrir durant cette phase 3 . 
L'étude comparative des variantes confinement / confiner et déconfinement / déconfiner (cf. Annexe 2) permet de confirmer cette tendance: les discussions relatives au confinement ont atteint un pic (plus de 1.200 citations par semaine) au moment de la première annonce des mesures (mi-mars), pour ensuite décroitre au fil des assouplissements de celles-ci. Les premières occurrences de déconfinement, elles, sont apparues dès la dernière semaine de mars, montrant sans doute un espoir de sortie de la crise, pour ensuite augmenter et atteindre un pic fin avril lorsque le pouvoir politique a, lui-même, abordé les possibles premières mesures le mois suivant. Les courbes des occurrences des deux termes finissent par se croiser au moment de la phase 3 du déconfinement (cf. supra).

(12) Rendez-vous est donné la semaine prochaine pour une communication du conseil de sécurité sur la stratégie de déconfinement en plusieurs phases basées sur les avis des experts. D'ici là, on ne lâche rien! \#RestezChezVous \#Covid19BE \#coronavirus (15 avril 2020)

L'analyse en constituants immédiats des tweets belges a permis d'approfondir le rôle que le Covid-19 joue dans les représentations de la population, notamment en tant qu' "acteur responsable" des événements en cours. II est intéressant de noter que les expressions relatives au Covid-19 sont sujets de phrases actives dans seulement $2,8 \%$ des cas (2.963 sur 107.305$)$. L'analyse qualitative de ces emplois "actifs" du virus dans les tweets des personnalités politiques permet une spécification thématique du Covid-19, tel qu'il veut être représenté au niveau politique. On retiendra les 4 catégories thématiques principales: (1) les comportements du Covid-19 et leurs retombées dans la société (exemple 13); (2) l'humanisation du virus, qui revêt une conscience, des connaissances et une volonté propre (exemple 14); (3) Les métaphores du flux, de la vague et de l'eau, déjà mises en avant pour d'autres crises de société, telles que les crises climatique et migratoire (Neagu \& Colipcă-Ciobanu 2014): le virus s'échappe, s'enfuit, circule, inonde et migre (exemple 15); (4) les dimensions sanitaires d'un virus (exemple 16).

(13) Le Corona virus a enlevé le masque du capitalisme: la norme doit être sociale et écologique. (1 ${ }^{\text {er }}$ mai 2020)

(14) Italica, une Italienne de 102 ans, guérie du Covid-19: "Face à elle, le coronavirus a abandonné" (29 mars 2020)

(15) Coronavirus et déconfinement en Belgique: voici où le Covid-19 circule le plus depuis la réouverture des magasins le 11 mai! (31 mai 2020)

(16) Le coronavirus n’a pas contaminé la combativité des métallos italiens (20 avril 2020)

Au-delà de la dynamique passive-active du virus dans les expressions utilisées sur Twitter, nous nous sommes aussi penchés sur les lemmes les plus associés au Covid-19 dans les tweets des Belges francophones. Une analyse part-ofspeech (POS) de ceux-ci a permis de dresser un classement des 20 lemmes les plus fréquents par catégorie grammaticale (Annexe 3 ). Les substantifs les 
plus fréquemment employés dans l'environnement relatif au Covid-19 revêtent principalement une connotation négative, relevant les conséquences négatives de la pandémie: crise (6.558 occurrences), décès (3.906), mort (3.410) et patient (2.170) recouvrent à eux-seuls un quart (24\%) des occurrences de cette liste. Une deuxième fraction de cette liste de substantifs se révèle plus neutre, décrivant le virus (épidémie, pandémie et virus), et les événements (mesure, confinement), lieux (hôpital, pays, monde) et objets (masque, test) relatifs au coronavirus. Notons que les descripteurs liés aux tweets sur le Covid-19 relèvent principalement de référents concrets, ce qui, selon Borden \& Zhang (2019), est souvent indicateur d'une faible attribution de la responsabilité de la crise aux acteurs de société. Nous relevons tout de même deux unités plus abstraites: les cas et la santé.

(17) Un cas suspect de coronavirus à Liège (29 février 2020)

(18) Des cas atypiques de coronavirus inquiètent l'OMS (22 février 2020)

(19) La Belgique passe en phase 2. Avec les retours de vacances (de Carnaval), il faut s'attendre à voir émerger d'autres cas dans le courant de ces prochains jours (1 ${ }^{\text {er }}$ mars 2020)

(20) La Belgique annonce 31 nouveaux cas de coronavirus: un total de 200 personnes contaminées (8 mars 2020)

Pour ce qui est de cas, les exemples 17 à 20 illustrent l'approximation liée à l'utilisation de ce mot: tantôt individu "candidat" au Covid-19, tantôt "diagnostiqué" Covid-19, tantôt simplement "contaminé" par le coronavirus. Pour ce qui est de santé, c'est une analyse plus fine de ses concordances qui nous permet de comprendre son utilisation en contexte. Celles-ci, illustrées en Annexe 4, mettent en avant le fait que le terme "santé" n'est presque jamais utilisé seul, mais toujours au sein d'expressions aux signifiants très variés. Parmi ces expressions, on retrouve: santé publique (250), soins/services de santé (224), professionnels/travailleurs de la santé (106), système de santé (98), bonne santé (83), santé mentale (53), secteur(s) de la santé (39), personnel de santé (19), commission santé (18), santé de tous (17) et santé financière (8).

Contrairement aux substantifs, les formes verbales (dont on a exclu les verbes supports) et adjectivales associées au Covid-19 semblent revêtir une connotation plutôt positive, tenant de l'appel à l'entraide et à la solidarité (aider, soutenir) et de l'optimisme (bon, positif, possible). Les adjectifs les plus courants relèvent également du caractère novateur de la situation: nouveau (6.740 occurrences, exemple 21), premier (3.624) et autre (2.528).

(21) Coronavirus: un forfait pour éviter un temps d'attente trop long pour les nouveaux chômeurs temporaires (18 mars 2020)

II semble ressortir de ces analyses une idée prépondérante de collaboration, de travail et de soutien de groupe: c'était déjà le cas dans les expressions relatives 
à la santé (santé publique, santé de tous), cela ressort dans les verbes de solidarité (soutenir) et dans une série d'adjectifs qui mettent en avant le caractère communautaire de la problématique de la crise, comme européen (2.015 occurrences), social (1.459), public (1.021) et mondial (1.006).

\section{Personnalités du confinement}

La crise du Covid-19 a mis en avant le rôle particulièrement important de différents organismes et personnalités. D'une part, les personnalités politiques ont endossé le rôle de "managers de la crise" stricto sensu (Hearit \& Courtright 2004: 211). D'autre part, les médias dont nous avons vu précédemment (cf. partie 2.1.3) qu'ils s'avèrent essentiels dans la transmission de l'information, peuvent être considérés comme co-managers de la crise.

\subsection{Personnalités politiques}

Si l'attribution de l'origine de la crise du Covid-19 en Belgique a majoritairement

\begin{tabular}{llrrlr} 
Pos & \multicolumn{1}{c}{ Nom } & $\begin{array}{c}\text { Nb } \\
\text { tweets }\end{array}$ & Pos & \multicolumn{1}{c}{ Nom } & $\begin{array}{c}\text { Nb } \\
\text { tweets }\end{array}$ \\
$\mathbf{1}$ & Sophie Wilmès (MR) & 951 & $\mathbf{9}$ & Rudi Vervoort (PS) & 88 \\
$\mathbf{2}$ & Catherine Fonck (cdH) & 325 & $\mathbf{1 0}$ & François Desquesnes (cdH) & 79 \\
$\mathbf{3}$ & Christie Morreale (PS) & 273 & $\mathbf{1 1}$ & Sofie Merckx (PTB) & 78 \\
$\mathbf{4}$ & Georges-L Bouchez (MR) & 261 & $\mathbf{1 2}$ & Raoul Hedebouw (PTB) & 75 \\
$\mathbf{5}$ & Elio Di Rupo (PS) & 232 & $\mathbf{1 3}$ & Jean-Marc Nollet (ecolo) & 72 \\
$\mathbf{6}$ & Alain Maron (ecolo) & 168 & $\mathbf{1 4}$ & François De Smet (DéFI) & 66 \\
$\mathbf{7}$ & Willy Borsus (MR) & 136 & $\mathbf{1 5}$ & Denis Ducarme (MR) & 61 \\
$\mathbf{8}$ Caroline Désir (PS) & 93 & & &
\end{tabular}

été "externe" (l'alimentation chinoise, le pangolin, les chauve-souris...), l'attribution de la gestion de la crise a quant à elle été principalement dirigée vers les personnalités et organismes politiques. Dans les tweets relatifs au Covid-19, cela s'est traduit par un nombre très important d'interpellations des citoyens à l'égard des politiques, à travers les "mentions" (@).

Fig. 7 Classement des personnalités politiques francophones les plus citées et mentionnées (@) dans les tweets de la population belge francophone.

Les personnalités politiques francophones les plus mentionnées sont les responsables politiques aux commandes de la crise (la Première ministre Sophie Wilmès, le Ministre-Président wallon Elio Di Rupo), les ministres de la santé (Christie Morreale, Alain Maron) et les présidents ou porte-paroles de partis (Georges-Louis Bouchez, Raoul Hedebouw). Le cas de Catherine Fonck 
est atypique dans la mesure où elle est citée pour sa double-casquette de parlementaire et d'experte, médecin néphrologue.

(22) Coronavirus en Belgique: Catherine Fonck, au bord des larmes, partage le témoignage "de la détresse des soignants" (19 mars 2020)

Si on compare ce classement à celui des personnalités politiques les plus prolixes à propos du Covid-19 (Fig. 2), on observe que ce ne sont pas les comptes les plus présents sur Twitter qui sont les plus mentionnés (voir exemples de Germain Mugemangango et de Willy Borsus). Comme l'illustre la Fig. 1, ce sont les partis PTB $(29,8 \%), \mathrm{cdH}(16,6 \%)$ et MR $(13,1 \%)$ qui sont les plus prolixes durant cette période. La place importante du MR (à la tête du gouvernement minoritaire de plein exercice ${ }^{15}$ qui s'est vu attribuer des pouvoirs spéciaux pour gérer la crise du Covid-19) ainsi que la position du parti d'opposition d'extrême gauche PTB expliquent leur nombre important de tweets. On notera par contre une faible participation aux discussions de la part du parti ecolo, alors qu'un parallèle a été largement alimenté entre changement climatique (590 occurrences dans les tweets citoyens) et crise sanitaire du Covid-19, par l'effet de la mondialisation (Ladner \& Giard 2020).

Nous nous sommes également intéressés aux éléments différenciateurs au sein du discours de chacun des partis. Pour cela, nous avons utilisé comme corpus de référence l'ensemble des tweets politiques, puis avons réalisé une analyse de spécificité pour chaque sous-corpus, correspondant aux tweets propres à chaque parti. II ressort de cette analyse (Annexe 5) que les partis se démarquent entre eux par des expressions reflétant leur idéologie et touchant leur public-cible, même en pleine crise du Covid-19: indépendant, pension et profession libérale (pour le MR), fortune, salaire et taxe (pour le PTB), aide alimentaire, proximité et prévention (pour le PS), ou encore patient et soignant (pour le $\mathrm{cdH}$ ). Le parti ecolo, ici encore, ne se démarque pas par l'utilisation de termes relatifs à l'enjeu climatique (hormis biodiversité).

Nous avons également étudié l'image que les personnalités majeures de la crise du Covid-19 renvoient auprès des citoyens, en nous focalisant sur la Première ministre, Sophie Wilmès, qui a dirigé les mesures de crise. Comme l'ont montré certains auteurs en fouille d'opinion, les émotions liées aux crises sont principalement de deux ordres: la sympathie et la colère (Hoste et al. 2016). Ainsi, nous avons décidé de classifier manuellement un corpus restreint de tweets en fonction de ces deux émotions, en recourant à un continuum de "négatif" (colère, -1) à "positif" (sympathie, +1), en passant par "neutre" (informationnel, 0). Ce corpus est constitué des tweets des citoyens belges francophones mentionnant la Première ministre. La fig. 14 en Annexe 6

15 Pour rappel, au moment où s'est déclenchée la crise internationale du Covid-19, la Belgique n'était pas encore parvenue à constituer un gouvernement à la suite des élections fédérales belges du 26 mai 2019. Un "gouvernement minoritaire de plein exercice" est donc mis en place 17 mars 2020. 
présente l'évolution de cette polarité à travers le temps, au plus fort de la crise. La première tendance qui en ressort est la relative stagnation autour d'une moyenne de 0: ceci est explicable par une quantité importante de tweets purement informatifs, qui peuvent en réalité servir l'image de Sophie Wilmès. En effet, une information claire et soutenue a tendance à avoir des effets positifs dans l'infodémie vécue en période de crise, (Lits et al. 2020: 29).

(23) Lors de la conférence de presse du Conseil national de sécurité mercredi, @SophieWilmès a assuré que notre pays faisait partie du haut du classement en matière de testing. C'est vrai, mais on revient de loin (7 mai 2020)

La seconde tendance qui est mise en lumière par ce graphique est la variabilité d'opinion qui suit les événements maitrisés (annonce du confinement ${ }^{16}$ ) ou non (scandales des masques ${ }^{17}$ ) par la Première ministre. On notera, que certaines mesures ont été appréciées par la population (e.g. l'ouverture à un silo de 4 personnes $^{18}$ qui lui vaut un regain de popularité de plus de points). L'appréciation moyenne de la Première ministre sur Twitter est donc plutôt positive, surtout lorsqu'on la compare à la polarité d'opinion d'autres personnalités politiques (Cougnon \& de Viron 2020).

\subsection{Les médias}

En tant que co-managers de la crise, les médias se sont efforcés de remplir le "vide informationnel" (Coombs 2012) créé par cette crise. Nous étudions le discours médiatique à travers 2 points succincts: son usage des émoticônes et son emploi du champ sémantique de la peur.

Le discours médiatique est d'ordinaire assez formel et, principalement à l'écrit, il s'écarte le moins possible des normes orthographique, syntaxique et stylistique, hormis par effet de style. Conséquemment, nous ne nous attendons pas à ce que les médias utilisent les émoticônes traditionnels, par exemple, pour représenter une émotion ou une attitude, alors qu'ils se doivent justement de rester objectifs et neutres (nous n'en trouvons d'ailleurs aucune trace dans le corpus médiatique). Nous avons donc observé l'utilisation de ces émoticônes là où nous ne les attendions pas, c'est-à-dire dans les tweets des médias. La Fig. 8 reprend les émoticônes les plus importantes ${ }^{19}$ employés durant la période de confinement.

\footnotetext{
16 Exemple: https://www.rtbf.be/info/belgique/detail_coronavirus-un-conseil-de-securite-a16h?id=10460292 (RTBF, 17 mars 2020)

17 Exemple: https://www.contrepoints.org/2020/05/09/371089-scandale-des-masques-en-franceet-en-belgique (Contrepoints, 9 mai 2020)

18 Exemple: https://www.sudinfo.be/id186667/article/2020-05-08/la-regle-des-quatre-personnesentre-en-vigueur-ce-dimanche-voici-les-reponses (Sudinfo, 8 mai 2020)

19 Pour quantifier cette importance, nous avons croisé la fréquence et la pertinence de chaque émoticône. Nous avons donc indiqué les émoticônes les plus fréquents (1-10) puis, parmi les suivants, ceux qui décrivaient la situation de Covid-19.
} 


\begin{tabular}{|c|c|c|c|c|c|c|c|}
\hline pos & émoticône & $\begin{array}{l}\text { référent en } \\
\text { contexte }\end{array}$ & nb & pos & émoticône & $\begin{array}{l}\text { référent en } \\
\text { contexte }\end{array}$ & nb \\
\hline 1 & 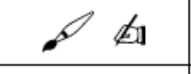 & rédigé par & 380 & 11 & مि & playlist/musique & 6 \\
\hline 2 & $\downarrow$ & info ici & 45 & 12 & $\Leftrightarrow$ & (corona)virus & 5 \\
\hline 3 & $\theta$ & masque & 25 & 13 & 曰 & télétravail & 5 \\
\hline 4 & (0) & alerte & 19 & 14 & (5) & soutien financier & 5 \\
\hline 5 & $\boldsymbol{w} \|$ & graphiques & 13 & 15 & 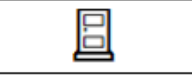 & confinement & 4 \\
\hline 6 & $t$ & voyages/avion & 12 & 16 & Vility & applaudissements & 4 \\
\hline 7 & $\Rightarrow$ & journal & 11 & 17 & إl|⿰口口 & culture / école & 4 \\
\hline 8 & $\Delta$ & attention & 9 & 18 & "4 & vaccin & 3 \\
\hline 9 & 曲 & magasins & 8 & 19 & †凮 & hôpitaux & 3 \\
\hline 10 & 象 & (corona)virus & 7 & 20 & 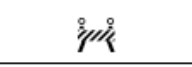 & barrière/distance & 2 \\
\hline
\end{tabular}

Fig. 8. Classement des émoticônes les plus importants dans les tweets des médias abordant la thématique du Covid-19 en Belgique francophone.

On y retrouve des émoticônes relatives à la pratique journalistique elle-même $(1,7)$, stipulant qu'il s'agit d'un article de journal ou en précisant l'auteur; les graphiques (5) sont également importants dans leur pratique, bien que cela ait été encouragé par les communications politiques elles-mêmes durant la crise. De même, on retrouve les émoticônes d'alerte $(4,8)$, qui attirent le regard du lecteur sur le tweet.

(24) \Attention aux "messages venant d'un ami médecin" qui prodiguent des remèdes contre le coronavirus partagés sur les réseaux. Nous avons vérifié, ces informations ne sont pas exactes. \#FakeNews \#coronavirus (15 mars 2020)

C'est la situation de confinement, et le virus lui-même, qui ont principalement fait l'objet d'une volonté de représentation imagée de la part des médias. Nous retrouvons la description du virus $(10,12)$, symbolisée par la double hélice d'ADN et un schéma de bactérie, ce qui rappelle la confusion qui existe entre virus et bactérie parmi les citoyens, qui se voit donc amplifiée par les médias. 
(25) Télétravail, indépendants, écoles... Tout ce que vous devez savoir sur le \#coronavirus (16 mars 2020)

On identifie également des représentations imagées des mesures $(3,15,20)$ et de la situation de confinement; parmi cette dernière catégorie, on différenciera les activités permises ou retreintes $(6,9,11,16)$ et les aides qui peuvent être apportées à la situation $(14,18,19)$.

(26) $\square \mathbf{Y}$ Avec les apéros virtuels, après le Covid-19, les hôpitaux vont avoir une épidémie de cirrhose. Note que l'origine est presque la même: c'est la Chine pour le premier et le tchin-tchin pour le deuxième (31 mars 2020)

(27) \#coronavirus | @AstraZeneca laisse entrevoir un vaccin \#Covid pour septembre (21 mai 2020)

On remarque que la fonction de ces émoticônes n'est pas lexicale et qu'elle tient davantage de l'accompagnement de propos, comme on a pu l'observer par ailleurs dans d'autres corpus pour souligner une émotion, par exemple (Cougnon, 2012). Or, dans le contexte médiatique, elles sont employées davantage pour annoncer la thématique générale du tweet et accrocher l'œil du lecteur que pour communiquer une émotion: les émoticônes sont utilisées majoritairement en début de tweet, annoncent ce qui va être énoncé par le contenu propositionnel et appuient la thématique principale.

Enfin, durant la crise du Covid-19, les médias ont été accusés de répandre la peur au sein de la population ${ }^{20}$. Ainsi, nous avons établi une ressource lexicale d'expressions relatives à la peur, que nous avons lemmatisées et recherchées parmi les tweets des médias durant la période de crise. La Fig. 15 en Annexe 7 présente une courbe qui dément cette assertion: en effet, si les médias se sont montrés inquiets $(8,5 \%$ des tweets comprenant un lexique relatif à la peur) à l'arrivée (mondiale) de la pandémie, ils se sont montrés plus rassurants avant même le début du confinement belge, la proportion de tweets issus du champ lexical de la peur ne dépassant plus 3\%.

\section{Conclusion}

Cette recherche exploratoire a permis de mettre en avant diverses conclusions relatives à la communication de la crise du Covid-19. Certaines de ces conclusions confirment les tendances observées lors d'autres crises majeures à travers le monde et l'Histoire, et certains résultats semblent tout à fait spécifiques à la pandémie de 2020. Pour rappel, nous désirions apprendre comment cette crise était nommée, à quoi elle se référait, et comment la population, les médias et les politiques communiquaient à son propos.

Les principales conclusions de cette étude sont les suivantes. L'appellation relative à la crise dans les tweets se confond avec l'appellation relative à la

20 Voir notamment https://www.lalibre.be/planete/sante/coronavirus-face-au-covid-19-les-belgesne-paniquent-pas-5e66221 ef20d5a29c64b24db. 
pandémie (coronavirus), mais, dans les tweets politiques, elle est en compétition avec la forme agglutinée covid19. Les formes plus complexes construites avec sras ou sars ne sont pas employées dans les échanges sur Twitter. En Belgique francophone, l'expression Covid-19 est de genre masculin. La période de crise a également vu l'émergence d'une néologie, qu'elle soit sémantique (confiner, confinement, distanciation sociale) ou lexicale (déconfiner, déconfinement). Le sujet du Covid-19 a abondamment alimenté les discussions de la population belge francophone, principalement au moment de la mise en confinement, qui a interpelé par son caractère soudain et insolite, suite à quoi, en moins d'un mois, le nombre de discussions sur le sujet a drastiquement décru (50\%). Les citoyens s'interrogent notamment sur les "comportements" du Covid-19 (humanisation, diffusion et retombées du virus). Le lexique employé dans les tweets de la population qui abordent le Covid-19 touche largement à l'entraide, la solidarité et le communautaire, en général. La population interpelle très souvent les personnalités politiques (Première ministre, ministres en charge de la santé), comme le permettent les "mentions" (@) de Twitter, qui en font un outil de dialogue direct entre les citoyens et le monde politique. Notre étude montre également que ce ne sont pas les comptes Twitter les plus productifs qui sont les plus interpelés, mais bien les comptes des acteurs majeurs de la crise. Les mentions (@ et urls) des médias dans les tweets des citoyens sont également importantes, surtout pour 3 comptes médiatiques (RTBFinfo, sudpresseonline et lesoir). De leur côté, les médias, en tant que co-managers de la crise, appuient l'information essentielle pour gérer la crise (notamment via le recours à des émoticônes spécialisées) et ne jouent pas le jeu de l'anxiété de foule (moins de 3\% de leurs tweets contiennent un lexique relatif à la peur).

Comme dans toute recherche exploratoire, les résultats exposés dans ce papier montrent leurs limites et nécessitent de nombreux autres développements. Tout d'abord, l'approche adoptée dans cette recherche est principalement monologique; il conviendrait d'étudier les aspects dialogiques des citoyens avec les managers de la crise. Ensuite, si certaines pistes de cette étude touchent aux émotions, nous souhaiterions approfondir leur étude, notamment à travers des méthodes de classification automatique de tweets. Enfin, nous avons pour projet de mener une étude comparatiste de nos résultats avec ceux des collègues français, suisses, luxembourgeois et italiens, pour offrir un panorama européen des tendances de communication.

\section{BIBLIOGRAPHIE}

Austin, L., Liu, B. F. \& Jin, Y. (2012). How Audiences Seek Out Crisis Information: Exploring the SocialMediated Crisis Communication Model. Journal of Applied Communication Research, 40:2, 188207. 
Borden, J., \& Zhang, X. A. (2019). Linguistic Crisis Prediction: An Integration of the Linguistic Category Model in Crisis Communication. Journal of Language and Social Psychology, 38(5-6), 650-679.

Coombs, T. (2012). Ongoing crisis communication: planning, managing, and responding (3e éd.). Thousand Oaks, California: SAGE.

Cougnon, L.-A. (2012). L'écrit sms. Variation en francophonie. Thèse de doctorat, dir. C. Fairon, Université catholique de Louvain.

Cougnon, L.-A. \& de Viron, L. (2020). Présentation Les Belges, le Covid-19 et les réseaux sociaux: 40 analyses durant le confinement. En ligne: https://uclouvain.be/fr/institutsrecherche/ilc/miil/reseaux-sociaux.html

Cougnon, L.-A. \& François, T. (2011). Étudier l'écrit SMS. Un objectif du projet sms4science. In A. Stähli, C. Dürscheid \& M.-J. Béguelin (éds), La communication par SMS en Suisse. Usages et variétés linguistiques. Linguistik Online (Themenheft).

Cougnon, L.-A., Roekhaut, S. \& Beaufort, R. (2013). Typologies de variation graphique dans l'écrit sms. In S. Baddeley, F. Jejcic \& C. Martinez (éds), L'Orthographe en quatre temps. 20e anniversaire des Rectifications de l'orthographe de 1990 : Enseignement, recherche et réforme, quelles convergences?. Honoré Champion.

Courtois, B. (2004). Dictionnaires électroniques DELAF anglais et français. In Ch. Leclère, É. Laporte, M. Piot \& M. Silberztein (éds). Lexique, Syntaxe et Lexique-Grammaire. Papers in Honour of Maurice Gross (pp.113-123). Amsterdam/Philadelphia: Benjamins, Lingvisticae Investigationes Supplementa 24.

de Caldas, S. (2015). Processus de création lexicale en français et en portugais contemporains dans le domaine de l'économie et de la finance. Revue française de linguistique appliquée, $x x(1), 45-$ 59.

Derczynski, L., Bontcheva, K. \& Roberts, I. (2016). Broad Twitter Corpus: A Diverse Named Entity Recognition Resource. Proceedings of the International Conference on Computational Linguistics (COLING).

Dumazedier, J. \& Ripert, A. (1966). Loisir et culture. Paris: Seuil.

Fediuk, T.A., Pace, K.M. \& Botero, I.C. (2010). Crisis Response Effectiveness: Methodological Considerations for Advancement in empirical Investigation into a Response Impact. In T. W. Coombs \& S. J. Holladay (éds), The Handbook of Crisis Communication (pp. 221-241). Malden, MA: Wiley-Blackwell.

Hearit, K. M., \& Courtright, J.L. (2004). A symbolic Approach to Crisis Management: Sears Defence of Its Auto Repair Policies. In D. P. Millar \& R. L. Heath (éds), Responding to Crisis. A Rhetorical Approach to Crisis Communication (pp. 201-213). Mahwah, New Jersey: Lawrence Erlbaum Associates, Publishers.

Holtzhousen, D. \& Zerfass, A. (éds) (2015). The Routledge Handbook of Strategic Communication. New York: Routledge.

Hoste, V., Van Hee, C., \& Poels, K. (2016). Towards a framework for the automatic detection of crisis emotions on social media : a corpus analysis of the tweets posted after the crash of Germanwings flight 9525. Proceedings of HUSO 2016. The Second International Conference on Human and Social Analytics (pp. 29-32). Barcelona, Spain.

Ladner, J. \& Giard, J. (2020). La pandémie COVID-19: une opportunité pour développer laformation en Santé Mondiale. Rapport publié sur le site de l'Agence universitaire de la Francophonie, https://actif.auf.org/wp-content/uploads/2019/10/une-opportunite-pour-developper-la-formationen-sante-mondiale.pdf

Larousse (2020). Dictionnaire de la langue française. En ligne: https://www.larousse.fr/dictionnaires/francais 
Lebart, L. \& Salem, A. (1994). Statistique textuelle. Paris: Dunod.

Lits, G., Cougnon, L., Heeren, A., Hanseeuw, B. \& Gurnet, N. (2020). Analyse de "l'infodémie" de Covid19 en Belgique francophone. ArXiv, https://doi.org/10.31235/osf.io/wsuj3

Lopez, C.E., Vasu, M. \& Gallemore, C. (2020). Understanding the perception of COVID-19 policies by mining a multilanguage Twitter dataset. ArXiv, https://arxiv.org/abs/2003.10359.

Neagu, M. \& Colipcă-Ciobanu, GI. (2014). Metaphor and self/other representations: A study on British and Romanian headlines on migration. In Musolff, A., Macarthur, F. \& Pagani, G. (éds). Metaphor and Intercultural Communication, pp. 186-201.

Rachfał, E. (2016). Towards a linguistic model of crisis response (CRModel). A study of crisis communication in the phone hacking scandal. Journal of Language and Politics, 15:2, 215-236.

Utz, S., Schultz, F. \& Glocka, S. (2013). Crisis communication online: How medium, crisis type and emotions affected public reactions in the Fukushima Daiichi nuclear disaster. Public Relations Review, 39:1, 40-46.

Veil, S. R. (2009). Friend or foe: Viewing the media as a partner in crisis response. Proceedings of the 12th Annual International Public Relations Research Conference, (pp. 667-674). Miami, FL: University of Miami.

Veil, S. R., Buehner, T. \& Palenchar, M. J. (2011). A work-in-process literature review: incorporating social media in risk and crisis communication. Journal of Contingencies and Crisis Management, 19:2, 110-122. 


\section{Annexes}

\section{Annexe 1}

\section{ROUVRIR VS RÉOUVRIR}

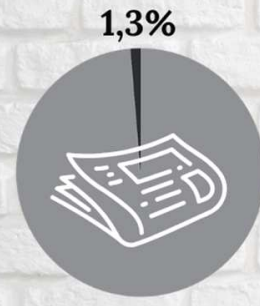

Médias
$8,5 \%$

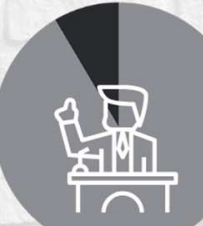

Politiques
$26,9 \%$

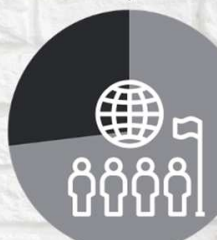

Population

Fig. 9 Analyse fréquentielle du verbe rouvrir et de sa variante réouvrir dans les tweets de la population, des personnalités politiques et des médias belges francophones (source: Cougnon \& de Viron 2020).

\section{Annexe 2}

CONFINEMENT VS DECONFINEMENT $\rightrightarrows$

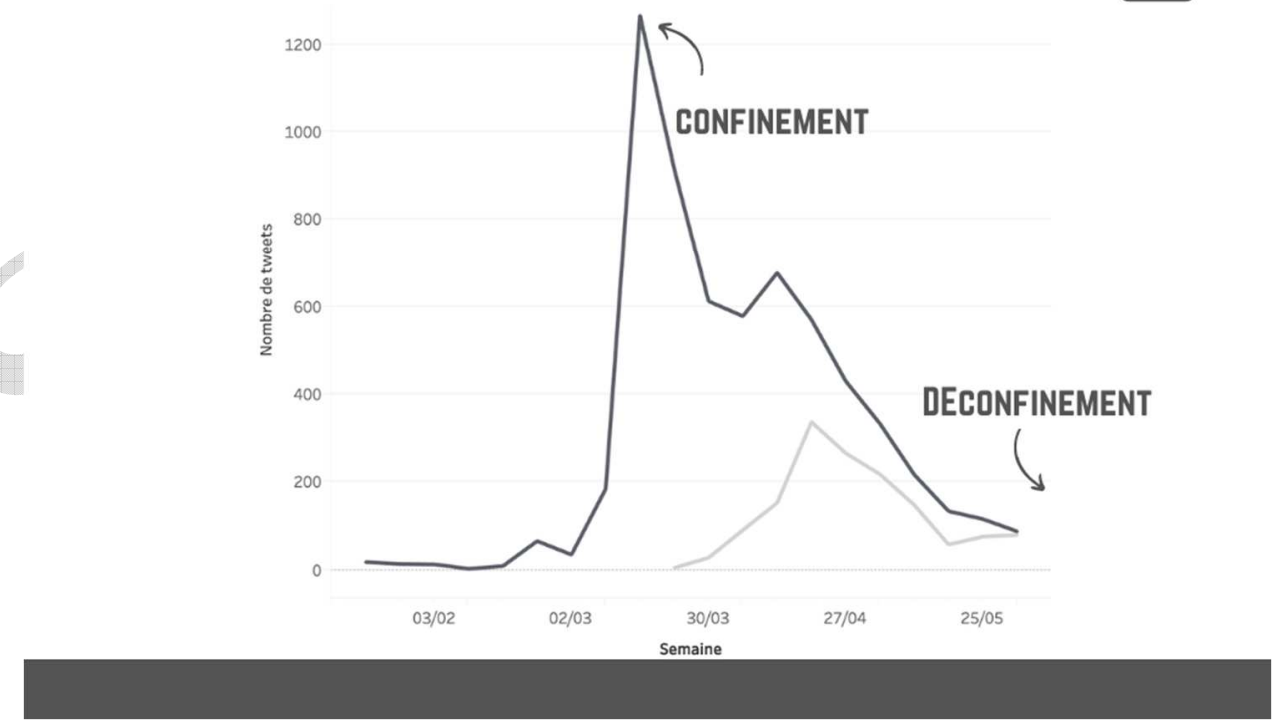

Fig. 10 Analyse fréquentielle de confinement/confiner et déconfinement/déconfiner dans les tweets de la population, des personnalités politiques et des médias belges francophones (source: Cougnon \& de Viron 2020). 


\section{Annexe 3}

\begin{tabular}{|c|c|c|c|c|c|}
\hline \multicolumn{2}{|c|}{ NOMS } & \multicolumn{2}{|c|}{ VERBES } & \multicolumn{2}{|c|}{ ADJECTIFS } \\
\hline lemme & tokens & lemme & tokens & lemme & tokens \\
\hline crise & 6558 & faire & 7521 & nouveau & 6740 \\
\hline cas & 5994 & avoir & 7259 & premier & 3624 \\
\hline mesure & 4769 & aller & 4848 & autre & 2528 \\
\hline confinement & 4730 & prendre & 3148 & européen & 2015 \\
\hline jour & 4510 & mettre & 2598 & grand & 1824 \\
\hline personne & 4189 & falloir & 2404 & bon & 1779 \\
\hline décès & 3906 & dire & 2332 & belge & 1713 \\
\hline mort & 3410 & voir & 1904 & positif & 1607 \\
\hline pays & 3028 & pouvoir & 1519 & social & 1459 \\
\hline monde & 2822 & savoir & 1515 & dernier & 1221 \\
\hline santé & 2744 & annoncer & 1333 & petit & 1171 \\
\hline hôpital & 2724 & suivre & 1145 & sanitaire & 1109 \\
\hline épidémie & 2723 & sortir & 1138 & économique & 1082 \\
\hline cause & 2463 & fermer & 1124 & total & 1072 \\
\hline pandémie & 2323 & lier & 1038 & public & 1021 \\
\hline test & 2246 & tester & 998 & mondial & 1006 \\
\hline patient & 2170 & annuler & 989 & chinois & 977 \\
\hline masque & 2107 & lutter & 971 & seul & 934 \\
\hline an & 2065 & aider & 968 & médical & 888 \\
\hline virus & 2035 & soutenir & 960 & possible & 781 \\
\hline
\end{tabular}

Fig. 11 Classement des unités lexicales les plus fréquemment associées à l'ensemble des tweets relatifs au Covid-19 (population, médias, politiques). 


\section{Annexe 4}

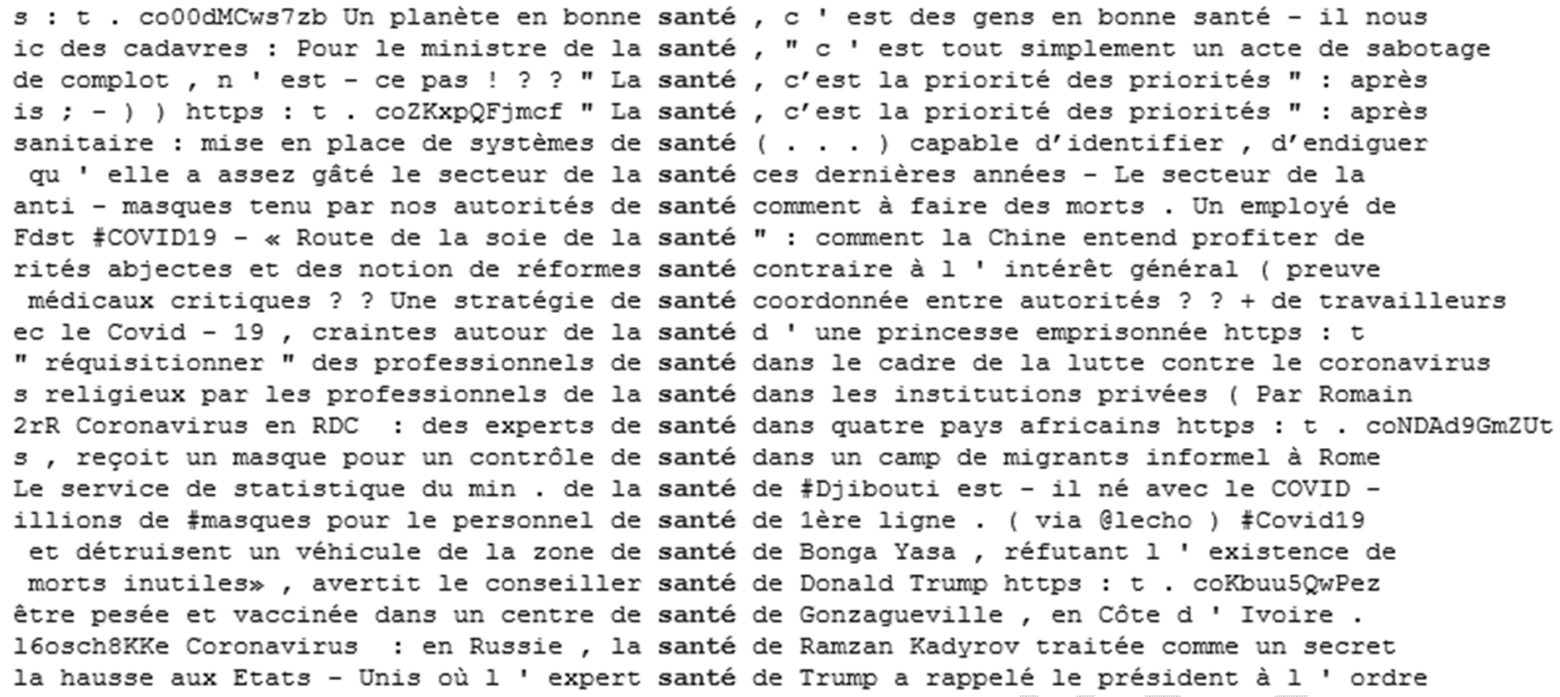

Fig. 12 Exemple de concordance avec le lemme "santé" dans le corpus de tweets relatif au Covid-19 en Belgique francophone.

\section{Annexe 5}

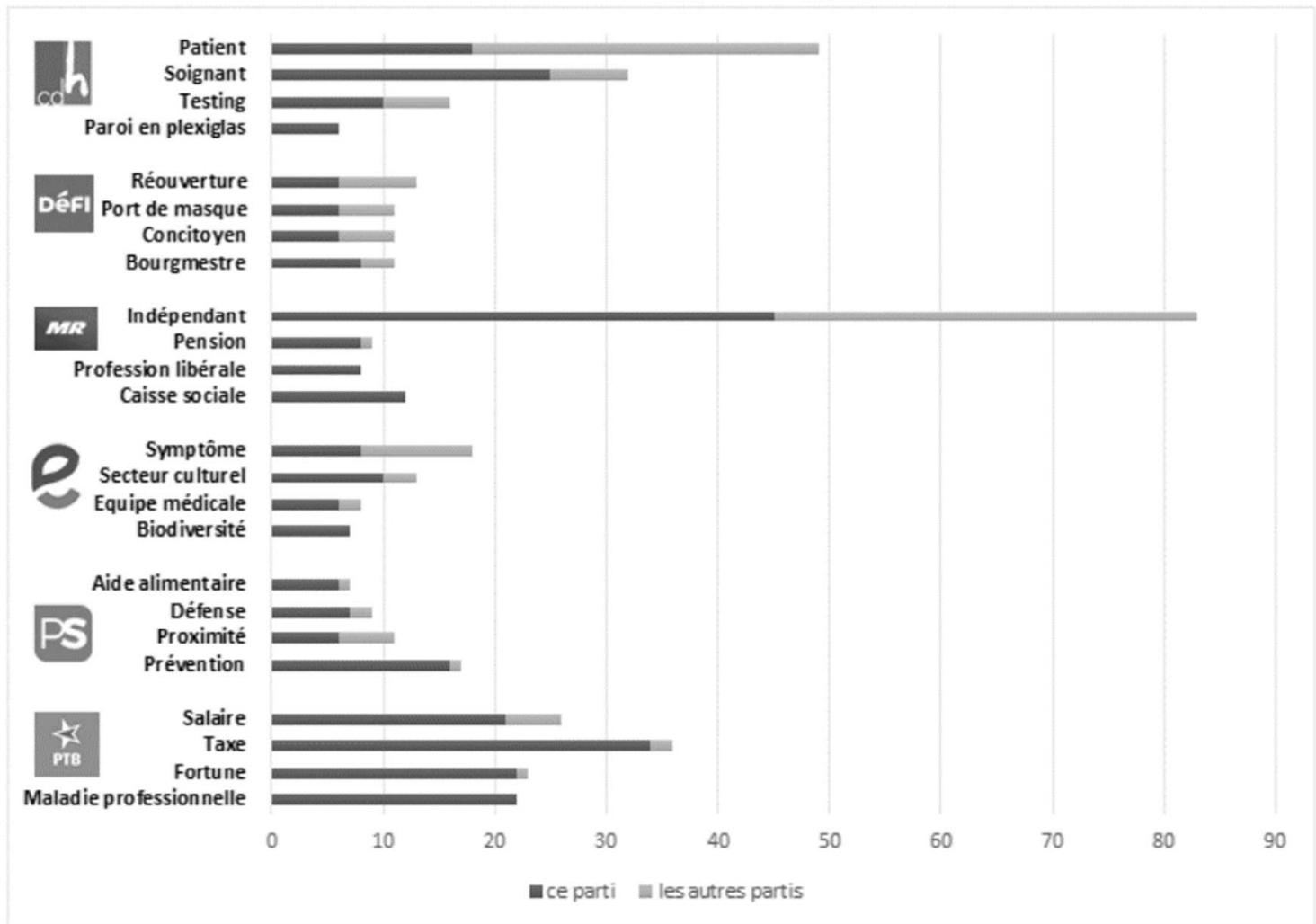

Fig. 13 Expressions spécifiques du discours politique belge francophone sur Twitter, par parti (source: Cougnon \& de Viron 2020). 


\section{Annexe 6}

\section{COMMENT VOYEZ-VOUS SOPHIE WILMES?}
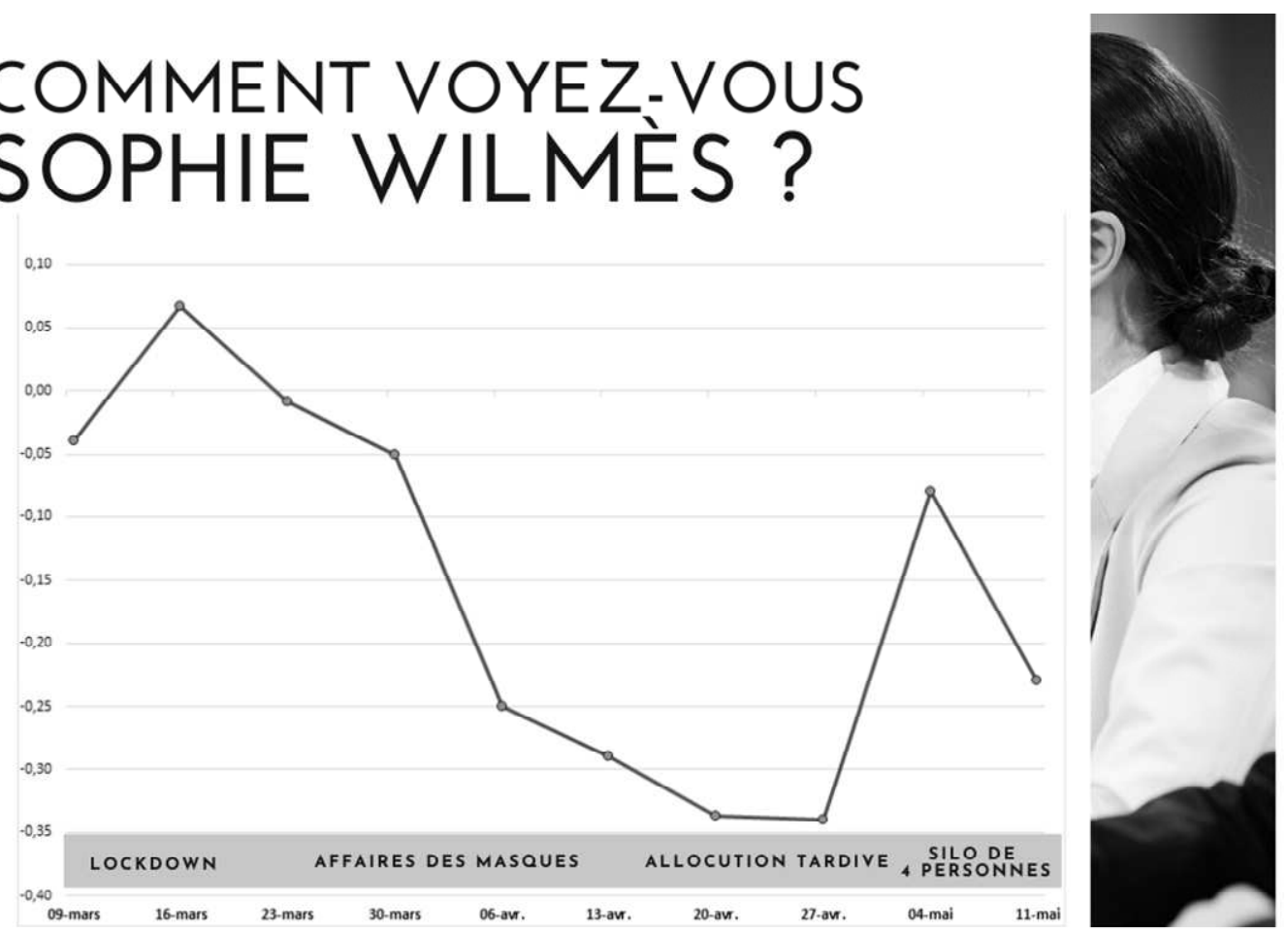

Fig. 14 Evolution de la polarité dans les tweets de la population qui mentionnent la Première ministre belge (source: Cougnon \& de Viron 2020).

\section{Annexe 7}

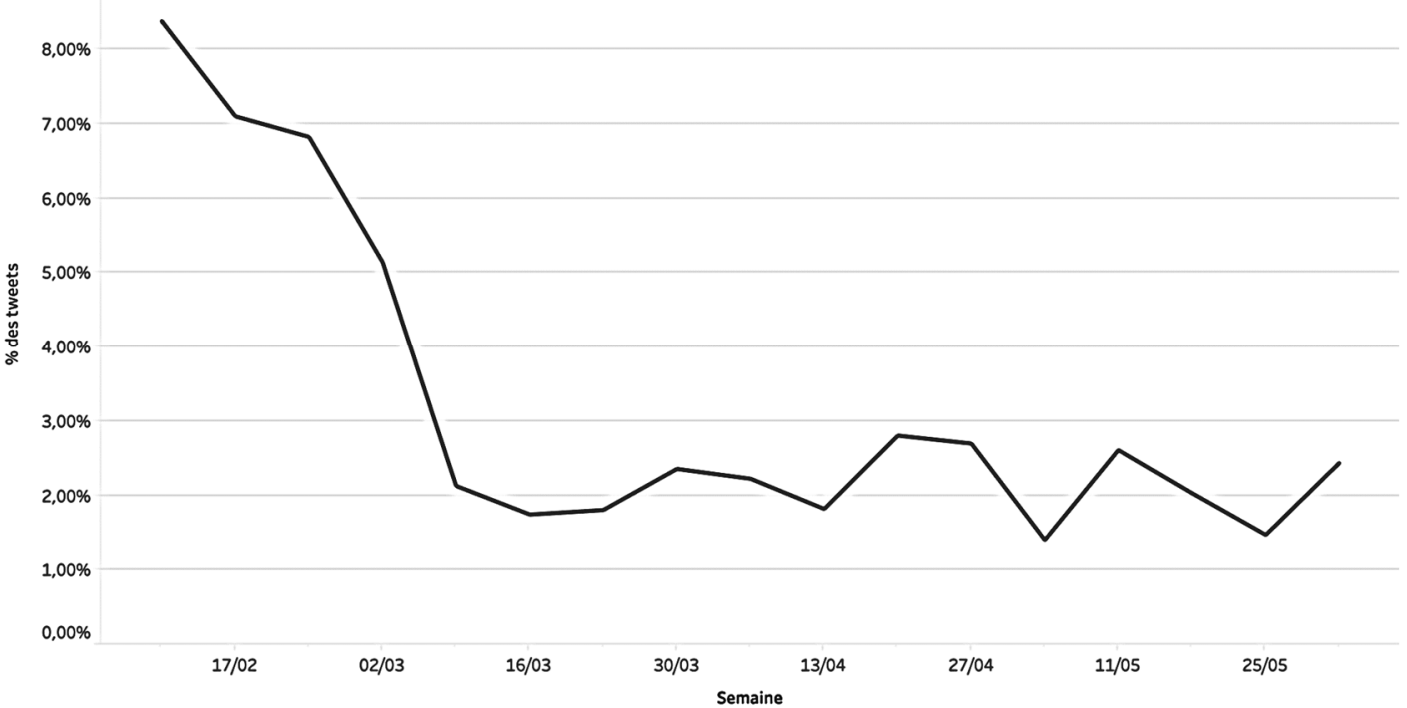

Fig. 15 Evolution du discours anxieux dans les tweets des médias belges francophones relayés sur Twitter. 